\title{
Gas-Nanoparticle Scattering: A Molecular View of Momentum Accommodation Function
}

\author{
Zhigang $\mathrm{Li}^{1}$ and Hai Wang ${ }^{2}$ \\ ${ }^{1}$ Department of Mechanical Engineering, University of Delaware, Newark, Delaware 19716, USA \\ ${ }^{2}$ Department of Aerospace and Mechanical Engineering, University of Southern California, Los Angeles, California 90089, USA
}

(Received 8 December 2004; published 30 June 2005)

\begin{abstract}
We examine the origin of diffuse gas-particle scattering by molecular dynamics simulation and show that diffuse scattering is the consequence of gas molecule trapping on the particle surface. Trapping occurs because of gas-particle interactions and the particle's ability of energy accommodation. These observations explain the transition from specular-to-diffuse scattering as the particle size becomes larger than the molecular size. We discuss the implication of this transition on the transport properties of nanometer-size particles.
\end{abstract}

DOI: 10.1103/PhysRevLett.95.014502

PACS numbers: $47.45 . \mathrm{Nd}$

Transport properties of nanoparticles in dilute gases are of great interest to nanoparticle synthesis, processing, and characterization, and the dispersion and monitoring of atmospheric ultrafine aerosol particles. These properties include electric and ion mobility, diffuse coefficient, drag, and thermophoretic velocity, and they are influenced by gas-particle momentum transfer. Traditionally, predictions of these properties are made with the StokesCunningham (SC) equation [1-3], where Cunningham's empirical slip correction was based on Millikan's experiments of oil droplets with radius $R>0.1 \mu \mathrm{m}$ [4]. The validity of this correction is highly questionable for the nanometer-size particle because in the molecular limit the SC equation does not converge to the Chapman-Enskog theory of molecular transport [5]. Previously, we developed a set of mathematical formulas for nanoparticle transport using the gas kinetic theory [6,7] and showed that the formulas are consistent with the Chapman-Enskog theory in the small-particle limit, and they also agree with the Epstein-Millikan formulation for micron-size particles $[4,8]$.

An interesting observation of our analysis is that a transition in collision dynamics must occur with particles a few nanometers in radius [7]. Below this size, gasparticle scattering is specular, and above this size the scattering is diffuse [9]. A similar observation was made by Tammet [10]. The existence of this transition may be inferred from Millikan's observation that gas scattering on micron-size oil droplets is mostly diffuse $[4,8]$ and by the success of the Chapman-Enskog theory [5], in which molecular scattering is assumed to be specular.

The two types of scattering give rise to different momentum accommodations [11], and in this way, they influence the transport properties of nanoparticles [6,7,12]. Diffuse scattering creates larger drag, smaller ion mobility, and diffusion velocity than do specular scattering. Although an analysis of available experimental data [1317] led us to suggest that the specular-to-diffuse transition occurs for a particle radius about $2.5 \mathrm{~nm}$ [7], close to the observation in $[10,18]$, little is known about what causes diffuse scattering and why the specular-to-diffuse transition occurs.

Here, we carry out molecular dynamics (MD) simulations to address these questions. Simulations are performed in the $(N+M, V, E)$ ensemble. We compute the collision dynamics of $M$ gas molecules $(M=1$ and 500) and a particle containing $N$ atoms. Here, we discuss results obtained with $M=1$ only, since the origin of diffuse scattering can be satisfactorily explained by single gas molecule-particle scattering, as will be discussed later. For convenience, the collision parameters are taken to be those of nitrogen $\left(\mathrm{N}_{2}\right)$ gas and the silver $(\mathrm{Ag})$ particle. The momentum accommodation of silver nanoparticles in air has been studied previously [7]. The simulations consider the thermal vibration of $\mathrm{Ag}$ atoms, employing a tightbinding potential function [19] widely used for predicting the dynamic behaviors of transition metals. In the current work, we predicted a melting point of $1275 \mathrm{~K}$ for bulk silver ( $1234 \mathrm{~K}$ experimentally) and the anticipated size dependence of the melting point, i.e., 375, 850, and $1000 \mathrm{~K}$ for particles $0.5,1.5$, and $3 \mathrm{~nm}$ in radius, respectively.

For $\mathrm{N}_{2}-\mathrm{Ag}$ atom pair interactions, we adopt the LennardJones $12-6$ potential function $\left(U=4 \varepsilon\left[(\sigma / r)^{12}-\right.\right.$ $\left.(\sigma / r)^{6}\right]$ ) and assume the total binding energy between $\mathrm{N}_{2}$ and the Ag particle to be a distance weighted sum between $\mathrm{N}_{2}$ and all Ag atoms in the particle. Here, $r$ is the separation distance, $\sigma$ the collision diameter, and $\varepsilon$ the well depth. An increase in the $\varepsilon$ effectively increases the gasparticle binding energy. The rotation of the $\mathrm{N}_{2}$ molecule inevitably leads to collision dynamics more complex than those assumed here. As will be discussed later, the qualitative conclusion that diffuse reflections are the result of molecular absorption is not affected by this complexity. Alternative gas-particle potential energy functions or methods to estimate these functions (e.g., [20]) may be necessary to obtain accurate particle radii of specular-todiffuse transition. This is not attempted here since there is no experimental data to directly verify the accuracy of the potential energy. For the same reason, we treat the well 
depth $\varepsilon / k$ as an adjustable variable, from 40 to $1000 \mathrm{~K}$, where $k$ is the Boltzmann constant. The collision diameter is chosen to be the arithmetic average of those for $\mathrm{N}_{2}$ and the Ag atom (see Table 4 of [7]).

Particles are constructed from an fcc silver crystal and by truncating atoms that lie outside of a sphere of radius $R$. The thus-defined particle is roughly spherical with a mass density equal to 9.6 to $10.6 \mathrm{~g} / \mathrm{cm}^{3}$, close to the bulk mass density. Ag atoms are initially assigned with an equal velocity corresponding to $300 \mathrm{~K}$, at directions generated from a random number generator. Integration of the equations of motion uses Beeman's leapfrog algorithm [21], with the time step equal to $1 \mathrm{fs}$. After 50 ps relaxation, collision is initiated by sending a $\mathrm{N}_{2}$ molecule towards the particle, initially spaced at one mean free path of the gas. A total of $160 \mathrm{MD}$ simulations were made, varying incident velocities and impact parameters $(0 \leq b<R)$ with or without particle rotation.

Atomic details and surface roughness are plausible causes for diffuse scattering, but since these details are shared among particles of all sizes, they are not sufficient to explain diffuse scattering. At ambient temperature particle rotation is too slow to smooth out the surface roughness. On the other hand, absorption, trapping, and desorption of a gas molecule on the particle surface can lead to a delayed reflection that is diffuse in nature. It follows that the specular-to-diffuse transition may be the consequence of increased molecular absorption on particle surfaces as the particle size and/or gas-particle binding energy increase. While evidence to support this hypothesis is abundant (see, e.g., [22,23]), a proof for this hypothesis has not been explicitly made.

We observe three types of collision dynamics. For a particle $1 \mathrm{~nm}$ in radius and a small gas-particle binding energy $(\varepsilon / k=211 \mathrm{~K})$ the gas molecule undergoes immediate reflections upon contact, as seen in the top panel of Fig. 1. The reflection is not exactly specular, but it is close to it. For the same particle and with a slightly larger $\varepsilon / k$ $(220 \mathrm{~K})$, the gas molecule becomes trapped on the particle surface, as seen in the middle panel of Fig. 1. The top panel of Fig. 2 shows that the translation energy of the gas molecule is absorbed by the particle upon contact, leading to gas trapping. The admolecule undergoes surface diffusion for $\sim 20 \mathrm{ps}$, about the same as the gas-particle collision frequency, during which time the admolecule undergoes surface diffusion and continuously exchanges energy with the particle. Because of energy fluctuation, the admolecule eventually regains energy just enough to desorb from the surface.

A further increase in the binding energy leads to "permanent" trapping within the simulation time period, ca. $75 \mathrm{ps}$, as seen in the bottom panel of Fig. 2. Here, the large binding energy, $\sim 10 \mathrm{kT}$, prevents the admolecule from desorption. This observation appears to be consistent with that of water-substrate interaction [23]. For $R=$
$1 \mathrm{~nm}, 300 \mathrm{~K}$ temperature, and $1 \mathrm{~atm}$ pressure, the gasparticle collision frequency is $\mathrm{O}\left(10^{11} \mathrm{~s}^{-1}\right)$. Therefore within the simulation time frame multiple molecules must have been absorbed. This should eventually reach an absorption-desorption equilibrium in which energy transfer is dominated by the collision of incoming gas
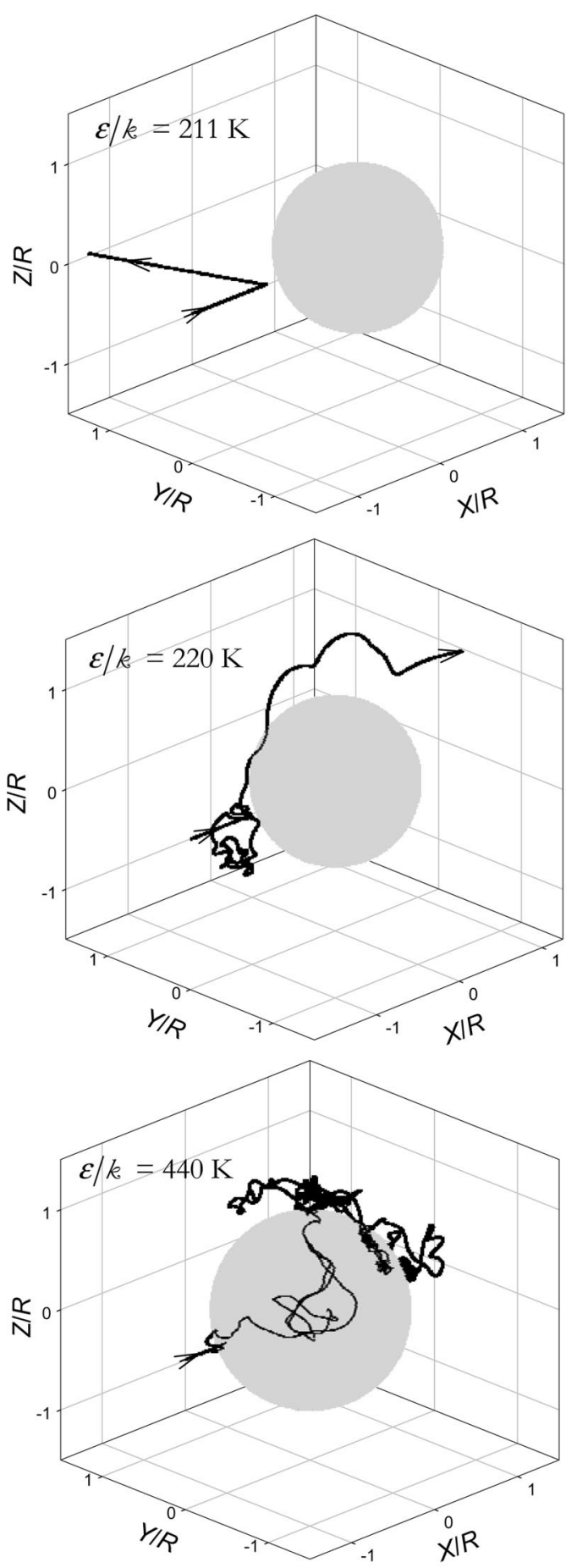

FIG. 1. Trajectories of the $\mathrm{N}_{2}$ molecule upon collision with a Ag particle $(R=1 \mathrm{~nm}$ and $b=0)$. The incident velocity corresponds to the mean relative velocity at $300 \mathrm{~K}$. 

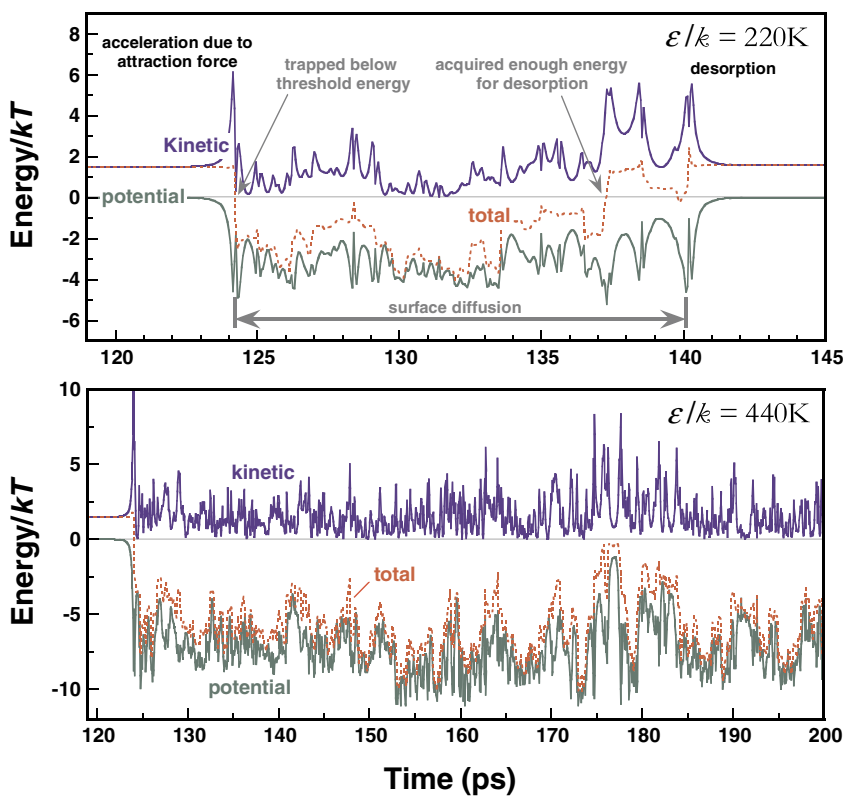

FIG. 2 (color online). Potential, kinetic, and total energies of the gas molecule. Computational conditions for the top and bottom panels are identical to those of the middle and bottom panels of Fig. 1, respectively. The $120 \mathrm{ps}$ is approximately the time the molecule takes to travel from one mean free path away from the particle.

molecules with the admolecules weakly bound to the $\mathrm{Ag}$ particle. These are confirmed by simulations with multiple gas molecules $(M=500)$.

Gas-particle scattering and especially gas trapping are dependent on surface curvature and the ability of energy accommodation by the particle, both of which are particlesize dependent. In Fig. 3, the critical $R$-vs- $k T / \varepsilon$ boundaries are drawn on the basis of MD simulations for the three scattering-absorption scenarios: immediate reflection, trapping desorption, and permanent absorption. For small particles $(R<1.5 \mathrm{~nm})$ at a given well depth (e.g., $k T / \varepsilon=3$ ) the reflections are immediate (and thus roughly specular), whereas for large particles $(R>1.5 \mathrm{~nm})$ the reflections are diffuse. Therefore, the MD results qualitatively explain the specular-to-diffuse transition from small to large particles.

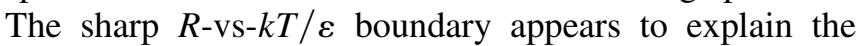
empirical observation that the specular-to-diffuse transition is quite sudden with respect to particle size [7], in spite of the underlying velocity distribution of gas molecules.

As expected, a higher temperature leads to a smaller absorption tendency. This effect is seen by the need to deepen the potential energy well to capture the gas molecule at large kinetic energies. Figure 3 shows that the effect of temperature scales with $\varepsilon / k$, and as such the $R$-vs- $k T / \varepsilon$ boundary is independent of temperature. This scaling suggests that at higher temperatures the specular-to-diffuse transition shifts to larger particle sizes, and the transition radius is smaller for larger well depth. Therefore, to predict the particle transition radius will require a fairly accurate

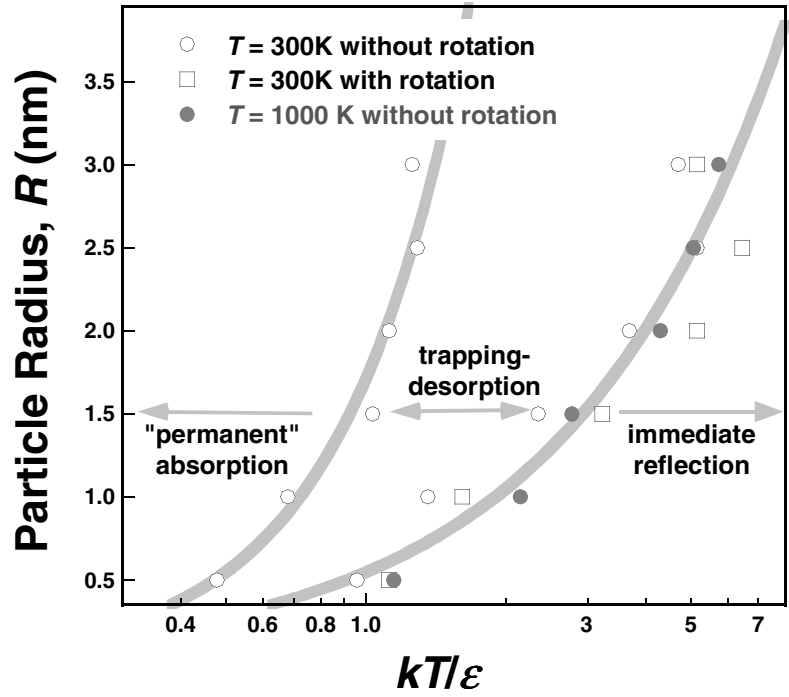

FIG. 3. $R$-vs- $k T / \varepsilon$ boundaries separating regions of specularlike, immediate reflections, diffuse scattering due to trapping or desorption and permanent absorption. Symbols are molecular dynamics "data" and lines are fits to the data. In the trappingdesorption region, the trapping probability is $\sim 90 \%$ for $0 \leq b \leq$ $R, \quad 1 \leq R \leq 6 \mathrm{~nm}$, and incident velocities $=0.8,1.0$, and 1.2 times the mean velocity.

potential function of gas-particle interactions, and, in particular, rotation of the fluid molecule may have to be considered. Taken together, the momentum accommodation is undoubtedly dependent on temperature, particle material, and the nature and composition of the gas in addition to particle size. On the other hand, the critical $R$-vs- $k T / \varepsilon$ boundary is not sensitive to particle rotation (see Fig. 3) and the impact parameter (not shown here).

We now comment on Epstein's idealized diffuse scattering model [9]. This model would be valid if the diffusion length is small compared to the particle size. Let us define here an absorption-trapping-desorption angle $\theta$ to be the central angle between the points of initial impact and final detachment (see the inset of Fig. 4). Here Epstein's model corresponds to $\theta \sim 0^{\circ}$, and, as a limiting case, we denote this limit as type I or hemisphere diffuse scattering. If the length of diffusion is comparable with the circumference of the particle, desorption leads to scattering into the entire sphere and $\theta$ should be widely distributed with its mean value equal to $90^{\circ}$. This second limiting case is referred to as type II or full-sphere diffuse scattering.

Figure 4 shows the $\theta$ values computed with $\varepsilon / k=$ $220 \mathrm{~K}$ and $T=300 \mathrm{~K}$, as a function of $R$. The variation of $\theta$ for a given particle size is the result of different particle orientations with respect to the incident gas molecule, the variations of the impact parameters $(b=0, R / 2$, and $R)$ and relative gas-particle velocities $(0.8,1.0$, and 1.2 times the mean velocity). Clearly, surface diffusion is nonlocalized for all particle sizes considered herein. Smaller particles $(R=1$ and $1.5 \mathrm{~nm})$ tend to give scatter- 


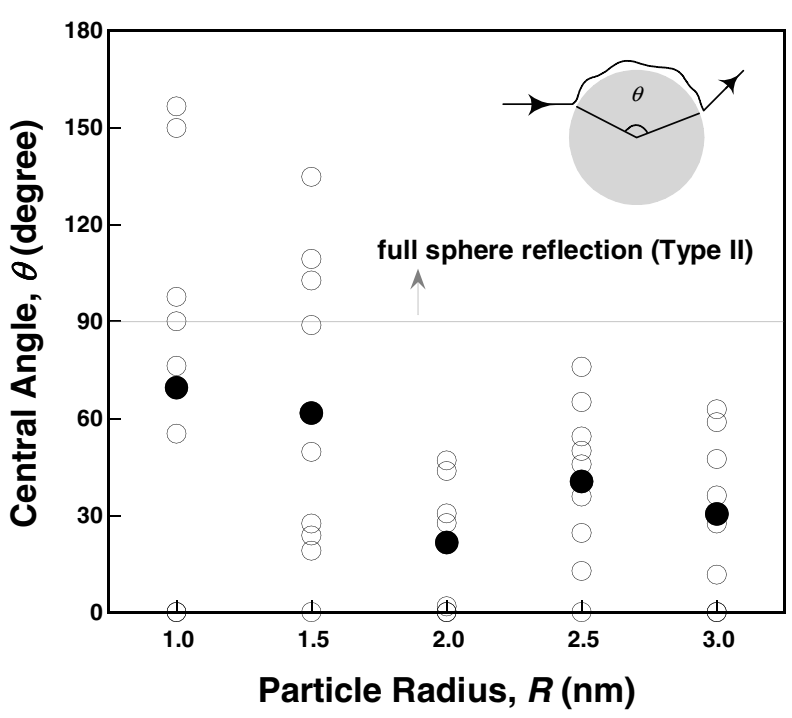

FIG. 4. Trapping-desorption angles computed (open circles) and averaged (filled circles) for $\varepsilon / k=220 \mathrm{~K}$ and $T=300 \mathrm{~K}$. The variation of $\theta$ for each $R$ is due to variations in the particle orientation with respect to the incidence, the impact parameter ( $b=0, R / 2$, and $R$ ), and relative gas-particle velocity $(0.8,1.0$, and 1.2 times the mean velocity). Cases with $\theta=0^{\circ}$ represent immediate reflections that occur $\sim 10 \%$ of the time.

ings closer to type II, whereas larger particles $(R \geq 2 \mathrm{~nm})$ give scatterings closer to type I. The above discussion led us to conclude that with an increase in the particle size, from small molecular clusters to micron-size particles, the collision dynamics evolve from direct, specularlike scattering, to diffuse scattering into the full sphere (type II), and finally to diffuse scattering into the hemisphere (type I). Because of finite lengths of surface diffusion, even micron-size particles must have some characteristics of type II diffuse scattering.

Using the analytical technique of [6], we obtain the collision cross section as $Q_{d-\mathrm{II}}=\pi b_{0}^{2}+2 \pi \int_{b_{0}}^{\infty}(1-$ $\cos \chi) b d b$ for type II diffuse scattering, where $b_{0}$ is the maximum impact parameter of physical contact, and $\chi$ is the angle of scattering for noncontact encounters. This cross section gives momentum accommodation values ranging from 0.1 to 0.4 for nonrigid body collision, depending on temperature, potential energy well depth, and particle size. Therefore, Millikan's observation of $90 \%$ type I diffuse and $10 \%$ specular scatterings for micron-size oil droplets [4] may well be explained by molecular absorption and finite-length surface diffusion.

Finally, we note that the particle transition radius is affected by the Knudsen number Kn, especially in the large Kn limit where the interactions of the fluid molecules impact the collision dynamics [24]. Amorphous, rough surfaces and the odd particle shape should also impact the transition radius, and these influences arise from geometric as well as potential energy variations.
We thank Professor Charlie Campbell, Professor Denis Phares, and Professor Anthony Wexler for helpful discussion and NSF for funding (CHE-0089136).

[1] E. Cunningham, Proc. R. Soc. A 83, 357 (1910).

[2] M. Knudsen and S. Weber, Ann. Phys. (Leipzig) 36, 981 (1911).

[3] M.D. Allen and O.G. Raabe, J. Aerosol Sci. 13, 537 (1982).

[4] R. A. Millikan, Phys. Rev. 22, 1 (1923).

[5] S. Chapman and T. G. Cowling, The Mathematical Theory of Non-Uniform Gases (Cambridge University Press, Cambridge, 1970).

[6] Z. Li and H. Wang, Phys. Rev. E 68, 061206 (2003).

[7] Z. Li and H. Wang, Phys. Rev. E 68, 061207 (2003).

[8] P. S. Epstein, Phys. Rev. 23, 710 (1924).

[9] Epstein's specular scattering [8] refers to molecular reflection whose angle is identical to the incidence. For diffuse scattering the gas molecule is scattered randomly into the hemisphere above the surface tangent to the particle at the initial contact.

[10] H. Tammet, J. Aerosol Sci. 26, 459 (1995).

[11] The momentum accommodation factor $\varphi$ is 0 and 1 in the specular and diffuse limits, respectively. Within the framework of Epstein's theory, the drag force may be expressed by $\mathbf{F}=-[(8+\pi \varphi) / 3] \sqrt{2 \pi m_{g} k T} N R^{2} \mathbf{V}[8]$, where $\mathbf{V}$ is the relative gas-particle velocity, $m_{g}$ the mass of the gas molecule, $k$ the Boltzmann constant, $T$ the temperature, $N$ the gas number density, and $R$ the particle radius.

[12] The drag force $\mathbf{F}$, diffusion coefficient $D$, and electric mobility in the limit of zero field strength $Z$ are bound by the same drag coefficient, $k_{d}$, viz., $\mathbf{F}=-k_{d} \mathbf{V}, D=$ $k T / k_{d}$, and $Z=q / k_{d}$, where $q$ is the charge in the particle. The $k_{d}$ value for diffuse scattering is larger than that of the specular model by $\sim 40 \%$ or greater [7].

[13] J. F. de la Mora, L. de Juan, K. Liedtke, and A. SchimidtOtt, J. Aerosol Sci. 34, 79 (2003).

[14] S. L. Kaufman et al., Anal. Chem. 68, 1895 (1996); 68, 3703 (1996).

[15] H. G. Scheibel and J. Porstendörfer, J. Aerosol Sci. 14, 113 (1983).

[16] Y. Kuga et al., J. Nanopart. Res. 3, 175 (2001).

[17] V. Ya. Rudyak and S. L. Krasnolutski, Dokl. Phys. 47, 758 (2002).

[18] M. Ramamurthi and P. K. Hopke, Health Phys. 56, 189 (1989).

[19] F. Cleri and V. Rosato, Phys. Rev. B 48, 22 (1993).

[20] J. Schulte, R. R. Lucchese, and W. H. Marlow, Z. Phys. D 26, S341 (1993).

[21] D. Beeman, J. Comput. Phys. 20, 130 (1976).

[22] R. Venkatesh, W. H. Marlow, R. R. Lucchese, and J. Schulte, J. Chem. Phys. 104, 9016 (1996).

[23] J. X. Fang, W. H. Marlow, J. X. Lu, and R. R. Lucchese, J. Chem. Phys. 107, 5212 (1997).

[24] E. B. Arkilic, K. S. Breuer, and M. A. Schmidt, J. Fluid Mech. 437, 29 (2001). 